\title{
Harnessing the neuroplastic potential of the human brain \& the future of cognitive rehabilitation
}

\author{
Jyoti Mishra* and Adam Gazzaley* \\ Department of Neurology, Physiology and Psychiatry, University of California, San Francisco, San Francisco, CA, USA \\ *Correspondence: jyoti.mishra@ucsf.edu; adam.gazzaley@ucsf.edu
}

Edited by:

Guido P. H. Band, Leiden University, Netherlands

Reviewed by:

Geert Van Boxtel, Tilburg University, Netherlands

Keywords: cognitive training, neurotherapeutics, cognitive control, neuroplasticity, closed loop

Neuroplasticity is the remarkable ability of the brain that allows us to learn and adapt to our environment. Many studies have now shown that plasticity is retained throughout the lifespan from infancy to very old age (Merzenich et al., 1991; Merzenich and DeCharms, 1996; Greenwood and Parasuraman, 2010; May, 2011; Bavelier et al., 2012). Enriching life experiences, including literacy, prolonged engagement in the arts, sciences and music, meditation and aerobic physical activities have all been shown to engender positive neuroplasticity that boosts cognitive function and/or prevents cognitive loss (Vance et al., 2010; Hayes et al., 2013; Matta Mello Portugal et al., 2013; Newberg et al., 2013; Zatorre, 2013). Unfortunately, just as enriching experiences generate positive plasticity, negative plasticity ensues in impoverished settings. For instance, many studies now show that low socioeconomic, resource-poor environments, which are associated with stress, violence and abuse within families and communities, have detrimental effects on cognition and neural function (D'Angiulli et al., 2012; McEwen and Morrison, 2013). As cognitive neuroscientists we observe both positive and negative aspects of plasticity in neural systems, in functional changes of neural activations, neural oscillations and strength of connectivity between brain regions, in structural changes in gray matter volume and white matter integrity, and importantly in the relationship between such neuroplastic changes and concomitant cognitive/behavioral changes. As we come to understand various facets of plasticity, it drives further the quest to develop new activities/interventions that engender maximal positive plasticity in selectively targeted neural systems; we envision such activities will in turn generate "far transfer of benefit" to generalized cognition and thereby improve the human condition.

In today's modern technological and internet-connected era, individuals are increasingly engaging with cognitive training software to improve cognitive function. In fact over the past 10-15 years, several companies have become established proponents and marketers of such software, transforming it into a multimillion dollar industry with exponential projected future growth. The fact that this technology is easily accessible over the internet to the home-setting, and at low-cost, has facilitated it's mass adoption. Scientifically, however, not all "brain training" is made equal. All too often, basic cognitive neuroscience experimental paradigms are embedded in commercial "brain training" approaches with add-on visual graphic skins that attempt to maximize user-engagement; a process known as gamification. Although these experimental paradigms had been originally developed to understand cognition, that does not mean that they are also the best tools to engender positive neuroplasticity. It is no surprise then that some scientific investigations have uncovered that generic brain training approaches yield no positive cognitive outcomes (Owen et al., 2010). However, a blanket statement that all cognitive training is ineffective is also unfair. In recent years, development and evaluation of cognitive training approaches in many labs, including our own, has revealed evidence for positive neuroplasticity, as well as for transfer of benefit to untrained cognitive abilities (Tallal et al., 1996; Temple et al., 2003; Stevens et al., 2008; Smith et al., 2009; Ball et al., 2010; Berry et al., 2010; Anderson et al., 2013; Anguera et al., 2013; Mishra et al., 2013; Wolinsky et al., 2013). Furthermore, in two of our training studies we find neurobehavioral correlations that relate on-task neuroplasticity to broader improvements in untrained aspects of cognition. Other researchers have also reported positive findings and transfer of training effects to untrained cognitive abilities in the context of custom-designed working memory exercises (Klingberg, 2010; Rutledge et al., 2012), task-switching training (Karbach and Kray, 2009), as well as for a specific genre of commercially available games, i.e., action video games (Bavelier et al., 2012) (although it is difficult to make strong recommendations about many off-the-shelf games given concerns over violent content). From these studies we are coming to understand some of the design principles that may govern the development of effective neuroplasticity-targeted training, as well as the scientific evaluation methods that can be used to provide convincing proof of the efficacy of the training intervention. Here, we summarize some of these principles that have emerged from two of our published training studies that now inform the development and evaluation of our next generation of training tools.

In our first training study in older adults, we simply trained visual perceptual discrimination of Gabor patches that had built-in directed motion animation (Berry et al., 2010). Ten hours 
of training improved on-task perception relative to performance changes in a non-training (no-contact) control group. Interestingly, the training also benefitted delayed-recognition working memory of an untrained motion direction task. Not only was working memory performance improved, electroencephalography (EEG) neural recordings showed that training evoked more efficient sensory encoding of the stimuli, which correlated with the working memory performance gains. This finding that $10 \mathrm{~h}$ of simple perceptual training engendered transfer of benefits to working memory aligns with recent understanding that perceptual training improves signal to noise contrast, which then leads to refined encoding at multiple neural scales and hence, at least some degree of generalized cognitive benefits (Vinogradov et al., 2012).

We are now gaining an appreciation that the observed gains in our perceptual training study, and in similar studies performed by other labs, some of which have shown long-lasting cognitive benefits (Willis et al., 2006; Rebok et al., 2014), may be mediated by two fundamental design elements that drive neuroplasticity. 1) Training incorporated continuous performance feedback at multiple levels of game play providing repeated cycles of reward to the user 2) Training was adaptive to the trainee's in-the-moment game performance; i.e., adaptivity was incorporated using psychophysical staircase functions that enhance training challenge in response to accurate performance and reduce it for inaccurate performance. The up-down step ratio in such staircases is often chosen to maintain overall task challenge at $75-85 \%$, at which point the user is optimally engaged but not frustrated. Thus, continuous performance feedback rewards and adaptive task challenge uniquely personalize the training to the cognitive capacity of each individual, and allows abilities to improve over time. Overall we have found these features to be critically important in generating positive neuroplasticity and cognitive benefit. Note, it is important to realize that casual game software is often not designed to provide the optimal dose of repetitive rewards nor incorporate adaptive progressions specifically targeted to the cognitive domains that may be deficient in a given population cohort. These factors, along with the heterogeneity of tested populations, very small training doses on multiple cognitive exercises, and the use of assessment measures that are insensitive to detect training related benefits in the tested population, all may contribute to a failure to observe positive impact (e.g., Owen et al., 2010).

While reward cycles and adaptive progressions are key components of software design, it is equally important to tailor these game mechanics toward improving specific deficits observed in a population cohort. For instance, Anguera et al. (2013) showed that deficient cognitive control abilities, such as working memory and sustained attention, in healthy older adults can be enhanced by specifically training on a multitasking performance-adaptive and rewarding video game, "Neuroracer." "Neuroracer" implements visual discrimination training in a go-no-go task for colored shape targets, with the added demand of simultaneously driving on a virtual road. "Neuroracer" evidenced extensive gains such that healthy older adults who multi-tasked $175 \%$ worse than younger adults on a first assessment, achieved significant post-training performance levels on the game itself that surpassed those of young adults by $+44 \%$. Importantly, training on "Neuroracer" transferred to untrained measures of sustained attention and working memory in the setting of interference, with EEG-based neural recordings showing that plasticity of midline frontal theta ( $\mathrm{mf}$ theta) neural oscillations may be a mediator of these cognitive improvements.

While we have tested some aspects of sound game design, as described, other aspects of high-level video games may contribute to their success and we look ahead to assessing these empirically. For example, immersion, fun, real-world features, continuous performance, 3D environments, virtual reality, high-levels of art, story, and music facilitate sustained performance and better compliance, and also deeper engagement that we suspect maximally harnesses plasticity. Evaluation of the influence of these features on training effectiveness requires careful scientific study design. For this, the "Neuroracer" study adopted a rigorous three-armed randomized controlled design. In addition to the multitasking training group, the study included an active single-task training control, as well as a no-contact control group. The singletask training control performed the exact same tasks as the training group of visual discrimination and driving, except that task engagement was not concurrent. This active control directly tested our hypothesis that only training in a setting that stresses cognitive control via a high interference environment would show significant cognitive gains. Outcomes of the "Neuroracer" multitasking training were not achieved in the active control group or in the no-contact group, the latter being critical for assessing practice effects due to repeated evaluations. Thus, the "Neuroracer" study highlighted that rigorous scientific evaluation of a cognitive training approach requires appropriate control groups, and often more than one control group, especially if we want to understand the underlying mechanisms of training effectiveness. Indeed longitudinal data collection is arduous, but without randomized, controlled and single/double blinded enrollments, we cannot convince ourselves of the significance of the results of new interventions. This is especially appropriate for healthy populations, while single-arm feasibility trials do remain informative as a first pass in cognitively impaired populations. In addition, we should also implement expectation bias measures for all participants, which confirm that all study groups anticipate the same level of influence of their assigned intervention on the outcome measures, thus assuring appropriate placebo control (Boot et al., 2013). Finally, adequately powered large sample size studies and investigations that measure sustainability of the cognitive gains and underlying neuroplasticity in yearly follow-ups are rare and need to be performed more often to address the long-term efficacy of cognitive interventions. Such rigor is convention in pharmaceutical clinical trials, and its adoption for video game testing, along with safety evaluations that detect potential side effects such as gameaddiction, would promote a path toward FDA approvals and medical prescription of such technologies.

Equipped with our growing understanding of how to design cognitive training approaches to target plasticity 
in specific neural circuits, we are now embarking on the development of the next generation training technologies. We envision these advances to include combining behavior-digital closed loops that link behavioral performance metrics to adaptive modulations of a training task on a digital platform, with neuro-digital closed loops that link neural performance measures to adaptive game mechanics. For example, the "Neuroracer" training study discovered that neuroplasticity of midline frontal theta (enhanced $\mathrm{mf}$ theta posttraining) is a key neural factor that correlates with transferred cognitive gains. In order to test whether mf theta plasticity is truly causal in enabling improved cognition, we are now developing neurodigital closed loops that directly target mf theta activity. More specifically, technological development is being directed at real-time EEG-based recordings that occur simultaneous with the cognitive task training (Delorme et al., 2011; Makeig et al., 2012; Kothe and Makeig, 2013). The goal is for these measurements to be event-locked to task stimuli, account for ocular and muscle-related artifacts, and use source localization algorithms (Mullen et al., 2013) so that they can be directly integrated in the game environment to guide reward feedback to the user and adaptivity of task challenge in real-time. We hypothesize that using neural performance as the driver for task-adaptivity will generate more rapid, efficient and specific circuit plasticity than is currently obtained using behavior-adaptive cognitive training approaches. This hypothesis is borne out of data, which shows that single-trial behavioral performance is predicted by neural measures such as mf-theta oscillations preceding the behavior. Thus the neuro-digital closed loop offers the potential to selectively train and refine the bottleneck neural processes that govern the final behavioral outcome. Importantly, by directly embedding taskrelated neural activity in a closed loop, this approach can provide missing causal evidence between neuroplasticity and cognitive benefits. This line of investigation is especially promising in the light of accumulating scientific evidence of the value of conventional neurofeedback approaches (Gruzelier, 2013; Wang and Hsieh, 2013; Arns et al., 2014), which also creates a neuro-digital closed loop, albeit driven by ongoing scalp EEG oscillations as opposed to task-related neural processes as we envision.

We are aware that unlike traditional cognitive training, a neuro-digital closed loop approach is not feasible as a mainstay in the home setting at present. Yet, with rapid developments of mobile EEG technology (Stopczynski et al., 2013), as well as advances in the real-time computational power available on consumer devices such as laptops and tablets, we expect that deployment in the home environment will be a reality within a few years. Neurodigital closed loops are also an exciting way to achieve personalized therapeutics, as each feedback loop is customized to the individual user's neural capacities in the moment. While here we have provided a simplistic example of a closed loop tied to task-related $\mathrm{mf}$ theta activation, one can conceive of more sophisticated neural targets, including frontal-posterior effective connectivity based on task interaction dynamics. Further advances in this field are expected as neuroscientists collaborate with neural engineers, who have predominantly focused related efforts on neuroprosthetic development (Borton et al., 2013). Neuro-engineers have designed efficient closed loop decoding algorithms for brain-machine interfaces in animal model systems, and these techniques are now ripe for adoption in humans (Carmena, 2013). Finally, especially beneficial for clinical populations that exhibit weakened neural responsivity, another intriguing step will be the integration of neuro-digital closed loop systems with transcranial electrical current stimulation or even deep brain stimulation technologies (Coffman et al., 2013), which may provide a needed plasticity boost to impaired brain regions.

To achieve the goals of our field and fully harness the potential of neuroplasticity for cognitive benefit, we look forward to continued technological development, such as neuro-digital closed loops, and their integration with emerging design principles of cognitive training games. These technologies validated using randomized, controlled scientific evaluation methodologies will generate new understanding of how to translate cognitive neuroscience discoveries into new educational tools for healthy populations and mental healthcare interventions for neuropsychiatric populations in need of cognitive remediation.

\section{ACKNOWLEDGMENTS}

This work was supported by the National Institute of Health grants 5R01AG040333 (Adam Gazzaley), 5R24TW007988-05 subaward VUMC38412 (Jyoti Mishra). We would like to thank Joaquin Anguera for his feedback on this opinion article.

\section{REFERENCES}

Anderson, S., White-Schwoch, T., Parbery-Clark, A., and Kraus, N. (2013). Reversal of agerelated neural timing delays with training. Proc. Natl. Acad. Sci. U.S.A. 110, 4357-4362. doi: $10.1073 /$ pnas. 1213555110

Anguera, J. A., Boccanfuso, J., Rintoul, J. L., AlHashimi, O., Faraji, F., Janowich, J., et al. (2013). Video game training enhances cognitive control in older adults. Nature 501, 97-101. doi: 10.1038 /nature 12486

Arns, M., Heinrich, H., and Strehl, U. (2014). Evaluation of neurofeedback in ADHD: The long and winding road. Biol. Psychol. 95, 108-115. doi: 10.1016/j.neubiorev.2013.09.015

Ball, K., Edwards, J. D., Ross, L. A., and McGwin, G. Jr. (2010). Cognitive training decreases motor vehicle collision involvement of older drivers. J Am Geriatr Soc, 58, 2107-2113. doi: 10.1111/j.15325415.2010.03138.x

Bavelier, D., Green, C. S., Pouget, A., and Schrater, P. (2012). Brain plasticity through the life span: learning to learn and action video games. Annu. Rev. Neurosci. 35, 391-416. doi: 10.1146/annurevneuro-060909-152832

Berry, A. S., Zanto, T. P., Clapp, W. C., Hardy, J. L., Delahunt, P. B., Mahncke, H. W., et al. (2010) The influence of perceptual training on working memory in older adults. PLoS ONE 5:e11537. doi: 10.1371/journal.pone.0011537

Boot, W. R., Simons, D. J., Stothart, C., and Stutts, C. (2013). The pervasive problem with placebos in psychology: why active control groups are not sufficient to rule out placebo effects. Perspect. Psychol. Sci. 8, 445-454. doi: 10.1177/1745691613491271

Borton, D., Micera, S., Millán, J. D. R., and Courtine, G. (2013). Personalized neuroprosthetics. Sci. Transl. Med. 5, 210rv2. doi: 10.1126/scitranslmed.3005968

Carmena, J. M. (2013). Advances in neuroprosthetic learning and control. PLoS Biol. 11, e1001561. doi: 10.1371/journal.pbio.1001561

Coffman, B. A., Clark, V. P., and Parasuraman, R. (2013). Battery powered thought: Enhancement of attention, learning, and memory in healthy adults using transcranial direct current stimulation. Neuroimage 85(Pt. 3), 895-908. doi: 10.1016/j.neuroimage.2013.07.083

D'Angiulli, A., Lipina, S. J., and Olesinska, A. (2012). Explicit and implicit issues in the developmental cognitive neuroscience of social inequality. Front. Hum. Neurosci. 6:254. doi: 10.3389/fnhum.2012.00254

Delorme, A., Mullen, T., Kothe, C., Akalin Acar, Z., Bigdely-Shamlo, N., Vankov, A., et al. (2011). 
EEGLAB, SIFT, NFT, BCILAB, and ERICA: new tools for advanced EEG processing. Comput. Intell. Neurosci. 2011, 130714. doi: 10.1155/2011/130714

Greenwood, P. M., and Parasuraman, R. (2010). Neuronal and cognitive plasticity: a neurocognitive framework for ameliorating cognitive aging. Front. Aging Neurosci. 2:150. doi: 10.3389/fnagi.2010.00150

Gruzelier, J. H. (2013). EEG-neurofeedback for optimising performance. I: a review of cognitive and affective outcome in healthy participants. Neurosci. Biobehav. Rev. doi: 10.1016/j.neubiorev.2013.09.015. [Epub ahead of print].

Hayes, S. M., Hayes, J. P., Cadden, M., and Verfaellie, M. (2013). A review of cardiorespiratory fitness-related neuroplasticity in the aging brain. Front. Aging Neurosci. 5:31. doi: 10.3389/fnagi.2013.00031

Karbach, J., and Kray, J. (2009). How useful is executive control training? Age differences in near and far transfer of task-switching training. Dev. Sci. 12, 978-990. doi: 10.1111/j.1467-7687.2009. 00846.x

Klingberg, T. (2010). Training and plasticity of working memory. Trends Cogn. Sci. 14, 317-324. doi: 10.1016/j.tics.2010.05.002

Kothe, C. A., and Makeig, S. (2013). BCILAB: a platform for brain-computer interface development. J. Neural Eng. 10, 056014. doi: 10.1088/17412560/10/5/056014

Makeig, S., Kothe, C., Mullen, T., BigdelyShamlo, N., and Kreutz-Delgado, K. (2012). Evolving signal processing for brain-computer interfaces. Proc. IEEE 100, 1567-1584. doi: 10.1109/JPROC.2012.2185009

Matta Mello Portugal, E., Cevada, T., Sobral Monteiro-Junior, R., Teixeira Guimarães, T., da Cruz Rubini, E., Lattari, E., et al. (2013). Neuroscience of exercise: from neurobiology mechanisms to mental health. Neuropsychobiology 68, 1-14. doi: 10.1159/000350946

May, A. (2011). Experience-dependent structural plasticity in the adult human brain. Trends Cogn. Sci. 15, 475-482. doi: 10.1016/j.tics.2011.08.002

McEwen, B. S., and Morrison, J. H. (2013). The brain on stress: vulnerability and plasticity of the prefrontal cortex over the life course. Neuron 79, 16-29. doi: 10.1016/j.neuron.2013.06.028

Merzenich, M. M., and DeCharms, R. (1996). "Neural representations, experience, and change," in The Mind-Brain Continuum, eds. R. Llinas and P. Churchland (Boston, MA: The MIT Press), 61-81.

Merzenich, M. M., Recanzone, G. H., and Jenkins, W. (1991). "How the brain functionally rewires itself," in Natural and Artificial Parallel Computations, eds. M. Arbib and J. A. Robinson (New York, NY: MIT Press).

Mishra, J., Anguera, J. A., Ziegler, D. A., and Gazzaley, A. (2013). A cognitive framework for understanding and improving interference resolution in the brain. Prog. Brain Res. 207, 351-377. doi: 10.1016/B978-0-444-63327-9.00013-8

Mullen, T., Kothe, C., Chi, Y. M., Ojeda, A., Kerth, T., Makeig, S., et al. (2013). Real-time modeling and $3 \mathrm{D}$ visualization of source dynamics and connectivity using wearable EEG. Conf. Proc. IEEE Eng. Med. Biol. Soc. Conf. 2013, 2184-2187. doi: 10.1109/EMBC.2013.6609968

Newberg, A. B., Serruya, M., Wintering, N., Moss, A. S., Reibel, D., and Monti, D. A. (2013). Meditation and neurodegenerative diseases. Ann. N.Y. Acad. Sci. 1307, 112-123. doi: 10.1111/nyas. 12187

Owen, A. M., Hampshire, A., Grahn, J. A., Stenton, R., Dajani, S., Burns, A. S., et al. (2010). Putting brain training to the test. Nature 465, 775-778. doi: 10.1038 /nature 09042

Rebok, G. W., Ball, K., Guey, L. T., Jones, R. N., Kim, H.-Y., King, J. W., et al. (2014). Ten-Year effects of the advanced cognitive training for independent and vital elderly cognitive training trial on cognition and everyday functioning in older adults. J. Am. Geriatr. Soc. 62, 16-24. doi: 10.1111/jgs. 12607

Rutledge, K. J., van den Bos, W., McClure, S. M., and Schweitzer, J. B. (2012). Training cognition in ADHD: current findings, borrowed concepts, and future directions. Neurotherapeutics 9, 542-558. doi: 10.1007/s13311-012-0134-9

Smith, G. E., Housen, P., Yaffe, K., Ruff, R., Kennison, R. F., Mahncke, H. W., et al. (2009). A cognitive training program based on principles of brain plasticity: results from the Improvement in Memory with Plasticity-based Adaptive Cognitive Training (IMPACT) study. J. Am. Geriatr. Soc. 57, 594-603. doi: 10.1111/j.1532-5415. 2008.02167

Stevens, C., Fanning, J., Coch, D., Sanders, L., and Neville, H. (2008). Neural mechanisms of selective auditory attention are enhanced by computerized training: electrophysiological evidence from language-impaired and typically developing children. Brain Res. 1205, 55-69. doi: 10.1016/j.brainres.2007.10.108

Stopczynski, A., Stahlhut, C., Petersen, M. K., Larsen, J. E., Jensen, C. F., Ivanova, M. G., et al. (2013). Smartphones as pocketable labs: Visions for mobile brain imaging and neurofeedback. Int. J. Psychophysiol. 91, 54-66. doi: 10.1016/j.ijpsycho.2013.08.007

Tallal, P., Miller, S. L., Bedi, G., Byma, G., Wang, X., Nagarajan, S. S., et al. (1996). Language comprehension in language-learning impaired children improved with acoustically modified speech. Science 271, 81-84. doi: 10.1126/science.271.5245.81

Temple, E., Deutsch, G. K., Poldrack, R. A., Miller, S. L., Tallal, P., Merzenich, M. M., et al. (2003). Neural deficits in children with dyslexia ameliorated by behavioral remediation: evidence from functional MRI. Proc. Natl. Acad. Sci. U.S.A. 100, 2860-2865. doi: 10.1073/pnas.0030098100
Vance, D. E., Roberson, A. J., McGuinness, T. M., and Fazeli, P. L. (2010). How neuroplasticity and cognitive reserve protect cognitive functioning. J. Psychosoc. Nurs. Ment. Health Serv. 48, 23-30. doi: 10.3928/02793695-20100302-01

Vinogradov, S., Fisher, M., and de Villers-Sidani, E. (2012). Cognitive training for impaired neural systems in neuropsychiatric illness. Neuropsychopharmacology 37, 43-76. doi: 10.1038/npp.2011.251

Wang, J.-R., and Hsieh, S. (2013). Neurofeedback training improves attention and working memory performance. Clin. Neurophysiol. 124, 2406-2420. doi: 10.1016/j.clinph.2013.05.020

Willis, S. L., Tennstedt, S. L., Marsiske, M., Ball, K., Elias, J., Koepke, K. M., et al. (2006). Longterm effects of cognitive training on everyday functional outcomes in older adults. J. Am. Med. Assoc. 296, 2805-2814. doi: 10.1001/jama.296. 23.2805

Wolinsky, F. D., Vander Weg, M. W., Howren, M. B., Jones, M. P., and Dotson, M. M. (2013). A randomized controlled trial of cognitive training using a visual speed of processing intervention in middle aged and older adults. PloS ONE 8:e61624. doi: 10.1371/journal.pone.0061624

Zatorre, R. J. (2013). Predispositions and plasticity in music and speech learning: neural correlates and implications. Science 342, 585-589. doi: $10.1126 /$ science. 1238414

Conflict of Interest Statement: Jyoti Mishra is a part-time scientist at the Brain Plasticity Institute, PositScience, a company that develops cognitive training software. Adam Gazzaley is co-founder and chief science advisor of Akili Interactive Labs, a company that develops cognitive training software. Jyoti Mishra and Adam Gazzaley have a patent pending for "Methods of Suppressing Irrelevant Stimuli." Adam Gazzaley has a patent pending for a gamebased cognitive training intervention: "Enhancing cognition in the presence of distraction and/or interruption."

Received: 30 December 2013; accepted: 27 March 2014; published online: 11 April 2014.

Citation: Mishra J and Gazzaley A (2014) Harnessing the neuroplastic potential of the human brain \& the future of cognitive rehabilitation. Front. Hum. Neurosci. 8:218. doi: 10.3389/fnhum.2014.00218

This article was submitted to the journal Frontiers in Human Neuroscience.

Copyright (c) 2014 Mishra and Gazzaley. This is an open-access article distributed under the terms of the Creative Commons Attribution License (CC BY). The use, distribution or reproduction in other forums is permitted, provided the original author(s) or licensor are credited and that the original publication in this journal is cited, in accordance with accepted academic practice. No use, distribution or reproduction is permitted which does not comply with these terms. 